\title{
Improving Human-Robot Interactivity for Tele-operated Industrial and Service Robot Applications
}

\author{
Yan Wu, Wei Liang Chan, Yanan Li, Keng Peng Tee, Rui Yan and Dilip K. Limbu
}

\begin{abstract}
In industrial robotics applications, teach pendant has been widely used by human operators to pre-define action trajectories for robot manipulators to execute as primitives. This hard-coding approach is only good for low-mix-highvolume jobs with sparse trajectory way-points. In this paper, we present a novel industrial robotic system designed for applications where human-robot interaction is key for efficient execution of actions such as high-mix-low-volume jobs. The proposed system comprises a robot manipulator that controls a tool (such as a soldering iron) to interact with the required workpiece, a networking server for remote tele-operation, and an integrated user interface that allows the human operator to better perceive the remote operation and to execute actions with greater ease. A user study is conducted to understand the merits of the proposed system. Results indicate that human can operate the system with ease and complete tasks more quickly and that the system can improve application efficiency.
\end{abstract}

\section{INTRODUCTION}

Since the first palletising robot was built in 1963, robots have started to assume an ever-increasingly important role in many industries. Traditionally, industrial robots are heavy automated machineries that are confined in a structured environment to perform fixed tasks at a high production volume predefined by human operators. Over the years, the way to programme these expensive robots have not evolved much due to the historical nature of the jobs in the production industry. Robots are expected to perform a relatively simple but highly repetitive task before the task changes. These lowmix-high-volume jobs are able to justify the robot cost using the economies of scale. However, in the past decade, the cost of robots including industrial robots has been driven down exponentially, making robots affordable to not only multi-national corporations but also small and medium-sized enterprises (SMEs). This demands robots to also handle highmix-low-volume (HMLV) production jobs which are typical for SMEs.

Conventionally, industrial robots are programmed through a teach pendant [1] in position control mode. A teach pendant can perform basic remote operations on the robot and record a time-series of waypoints for each individual degree-of-freedom (DoF). Programming robots using this time-consuming record-and-play method requires experienced end-users to hand-craft every robot step carefully. It is viable for relatively straight-forward robot motions which can be applied for a high volume production. However, this

Yan Wu, Wei Liang Chan, Yanan Li, Keng Peng Tee, Rui Yan and Dilip K. Limbu are with the A*STAR Institute for Infocomm Research, 1 Fusionopolis Way, 21-01 Connexis South, Singapore 138632. \{wuy, chanwl, liy, kptee, ryan, dklimbu\} at i2r.a-star.edu.sg programming overhead becomes counter-productive when the production volume is significantly reduced. Thus, the industrial robotics community is in need of a more intuitive way of operating the robot [2] to achieve higher efficiency for smaller volume mass production.

Robot programming by demonstration has been a popular topic of investigation for the past decade to allow non-expert end-users to teach robots new skills via demonstrations. It helps to reduce search space for the robots, teaching fatigue and time overheads for human demonstrators [3]. A human demonstrator interacts with the robot either physically or through a motion capture interface [4], [5]. Due to the physical constraints of industrial robots, it is impractical to programme these robots through physical human-robot interaction which is common in social robots [6], [7]. Thus, allowing the human operator to remotely control the robot to complete a task efficiently in the environment that is unfamiliar to the operator is key to bring industrial robots a step closer to HMLV jobs.

In this paper, we present the first prototype of our novel industrial robotic system which tackles the human-robot interactivity issue for HMLV jobs. This system comprises a robot manipulator that drives a tool to interact with the required workpiece, a networking server for remote teleoperation, and an integrated user interface that allows the human operator to better perceive the remote environment and operation. The action transmission unit of the user interface allows the user to execute actions with greater ease. A user study with 10 first-time users is conducted to understand the merits of the proposed system. Results indicate that human can operate the system with ease, the system enables operators to complete tasks more quickly and the system can improve application efficiency.

The rest of the paper is organised as follows: We will present the system overview and hardware specifications in Section II, followed by the control architecture and detailed implementations in Section III. The experiment design and analysis of results will be presented in Section IV before we conclude for future directions in Section V.

\section{System OVERVIEW}

The system comprises three main components: the robot and sensors at workpiece, the user control interface and the computation and networking services as shown in Fig. 1.

The system is designed to operate across a range of industrial robotic arms. In this work, we will use a 7DoF lightweight robot manipulator manufactured by KUKA (KUKA LBR iiwa 14 R820) which can more uniformly 


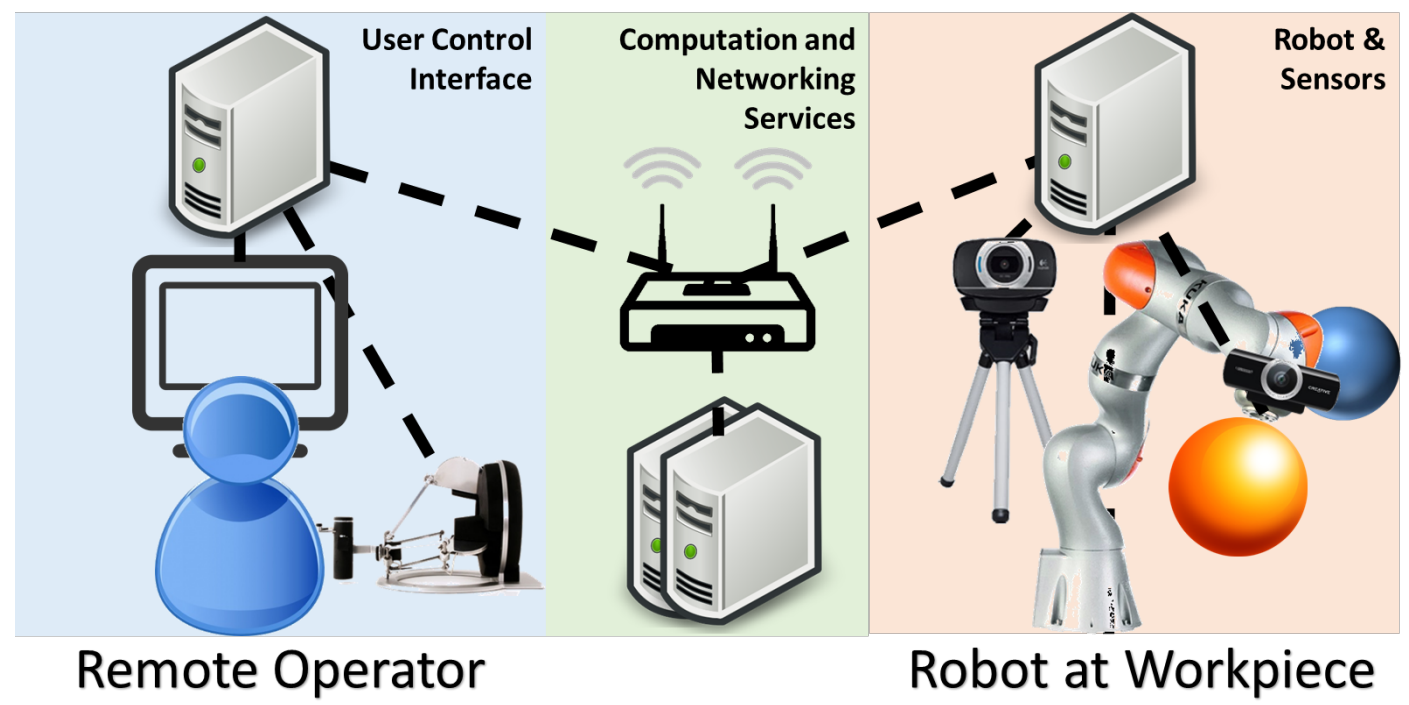

Fig. 1: The Overview of the System Setup

cover a larger workspace with high flexibility as compared to the traditional industrial robots. This robot is equipped with sensitive joint position sensors and joint torque sensors in addition to the conventional motor encoders at all joints for safe and compliant operations. Communications between KUKA LBR and its dedicated controller server, KUKA Sunrise Cabinet, is through its proprietary Fast Research Interface at the rate of $1 \mathrm{kHz}$. Apart from the typical global view camera placed in the workcell for the human operator to observe the robot's actions, we also introduce a local camera at the end-effector of the robot for real-time closeup view of workpiece being manipulated. Both cameras are Logitech C615 Webcams with full HD capability and short focal length of $7 \mathrm{~cm}$. The KUKA LBR, the KUKA Sunrise Cabinet which runs a Windows server and the cameras with USB2.0 are connected to a local PC for data communication with the remote operator, logging and processing.

At Remote Operator side, the human user can observe the robot through a dedicated large full HD monitor which displays the camera views side-by-side. The human operator controls the robot using the Omega-7 7-DoF haptic device (including $1 \mathrm{DoF}$ for gripper) developed by Force Dimension. The device provides up to $12 \mathrm{~N}$ of force and has a workspace of $110 \mathrm{~mm}$ depth and $160 \mathrm{~mm}$ diameter. The haptic device and the full HD display are connected to a local computer for data communication with the robot, logging and processing.

The two remote computers are connected via the Computation and Networking Services. It provides a dedicated TCP/IP and UDP communication link among computers in the network via a routing server. This includes the two remote computers and the server cluster for intensive data computation.

This system is designed to be intuitive for operation without much training. In the following sections, we will detail the implementation of the system.

\section{SySTEM IMPLEMENTATION}

The proposed system offers two modes of operations, the demonstration mode and the production mode. In the demonstration mode, the robot is controlled by the human operator to perform the task on the demonstration workpiece. The action executed by the human operator will then be processed and stored as a template of the task. In the production mode, the robot will use a morphable template approach [8] to batch reproduce the action template on a similar (not necessarily congruent) workpiece at a different pose.

Fig. 2 shows the schematic diagram of the process flow of the system. The demonstration mode is depicted in the yellow colour zone while the production mode is depicted in the blue colour zone. While the two local PCs handle the basic communications between the robot, router and sensors, the heavy computations are carried out in the computation servers.

\section{A. Demonstration Mode}

In the demonstration mode, there are three dependent input/output sources, namely the workpiece geometry readings, the haptic device setting/readings and the action template database. As perception is not the focus area of this work, we will illustrate how it can be used together with robot compliant control in our future work. In this work, we store the geometry of the workpiece in a file. It will be read at the start of a demonstration for correspondence mapping to the haptic device. The haptic device readings are read at the frequency of $1 \mathrm{kHz}$ and mapped to the robot workspace coordinates before being executed on the robot. Once an action demonstration is completed, the action trajectory is analysed and subsequently stored for use in the production mode in the action template storage.

1) Mapping between robot frame and haptic device: In this module, we implement two novel approaches to ease 


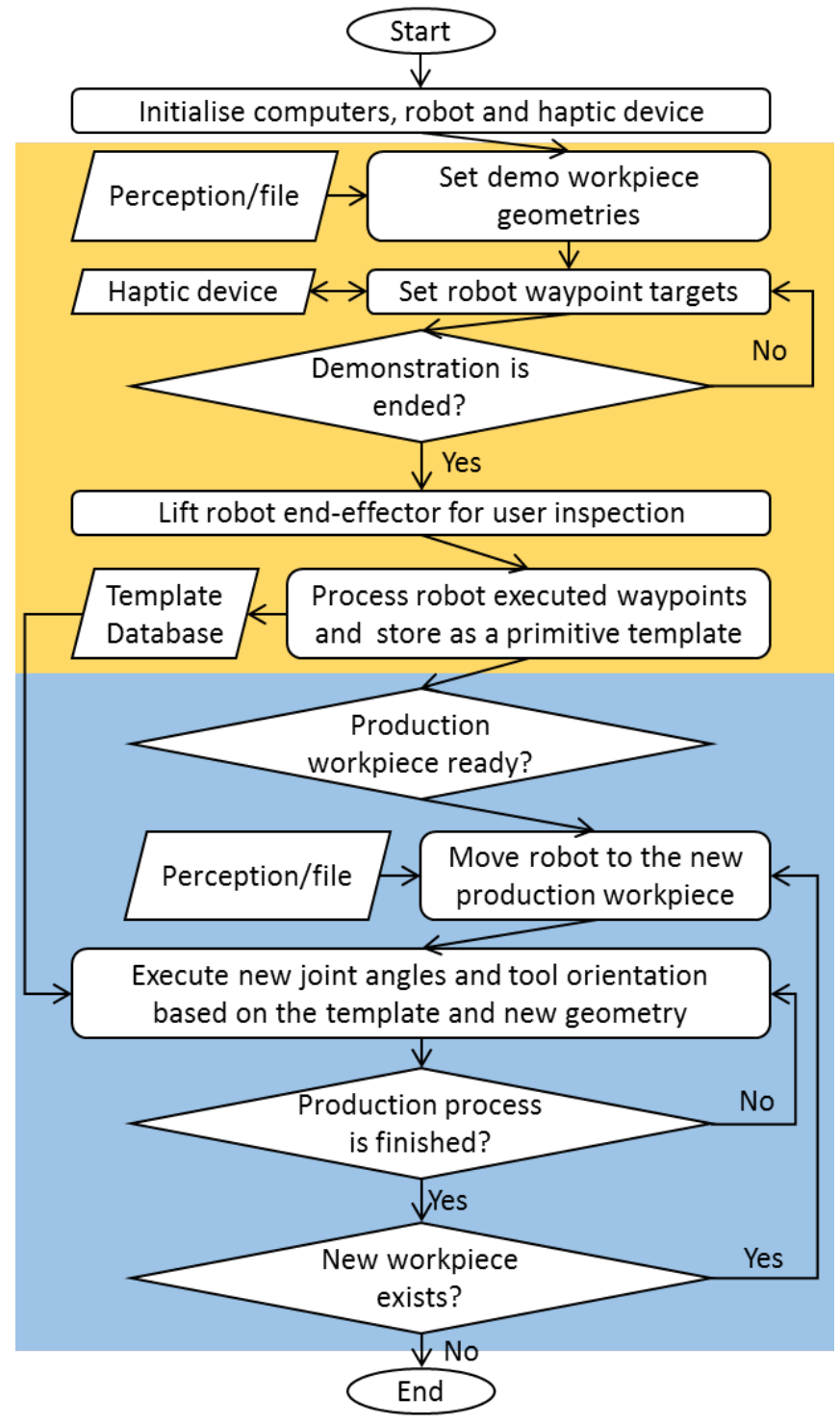

Fig. 2: The process flow schematics of the system. Yellow area depicts the demonstration mode while blue area depicts the production mode.

the problem of insufficient situatedness level for the remote operator and improve productivity.

Firstly, as the workpiece/object is not physically present in front of the operator, it is a difficult problem for the user to imagine a 3-dimensional (3D) object on the screen or around the haptic device. Instead of making the user construct an imaginary surface contour through interaction which is an inefficient and tedious process, the 3D surface of the workpiece geometry is morphed to a $2 \mathrm{D}$ plane and scaled to the workspace of the haptic device. This plane together with the force readings of the robot provides a virtual boundary/floor to provide feedback of force contact of the robot end-effector with the workpiece using high spring constant for workspace below the virtual 2D surface.

Secondly, in this application we introduce the concept of shared control [9], [10], [11]. Because of the low situation

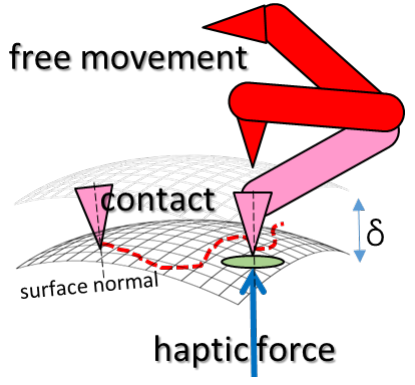

Fig. 3: Shared control in the demonstration mode. Robot takes control of supplementary DoFs and sets a buffer zone between the end-effector and the object surface. When the robot moves near the object (below the threshold $\delta$, robot controls the end-effector orientation. Otherwise, the human operator has the full control.

awareness in a remotely operated robot task, if the operator is given full access of the robot's 6-DoF workspace, he has to devote much of his effort to control the extra DoFs that ensure the successful completion of the task rather than the task action itself. For example, in a soldering task, the main action is to carry out the soldering at the required locations. The supplementary DoFs, such as the orientation of the soldering iron and the distance between the soldering iron and the object surface, are to ensure that the task can be completed successfully. And these supplementary DoFs are highly sensitive to perturbation and require high level of situation understanding. It is therefore beneficial for the robot to assume the task of controlling these supplementary DoFs while the human focuses on the main task especially when the robot's end-effector is near the surface of the workpiece. When the end-effector is away from the surface, the human operator can reclaim his full control of the robot arm. Thus, in this work we demonstrate the benefit of this hypothesis by implementing two shared control algorithms as shown in : 1) Robot takes care of the end-effector orientation with respect to the instantaneous surface of the workpiece (specified as a prior by the task); 2) Set a buffer threshold $\delta$ which ensures continuous contact between robot end-effector and the object aided by the haptic force experienced by the robot.

At each time step, once the pose of the robot end-effector is established, the inverse-kinematics is executed to map the workspace coordinate to joint space. As the KUKA LBR is a 7-DoF robot manipulator with a roll-pitch-yaw model that departs from convention, in order to avoid singularity of the on-board inverse kinematics (mapping from $6 \mathrm{DoFs}$ to $7 \mathrm{DoFs}$ ), we first choose a suitable roll value followed by a calculation of the pitch and then yaw values as illustrated in Code Listing 1.

A safety checking function is implemented to ensure no interpolated waypoints are inside the workpiece geometry. When the operator signals the end of a demonstration by pressing an escape button, robot then goes into a scripted programme in which it lifts the end-effector to position the local camera at a height that can capture the view of the entire 
workpiece. Once the user confirms the action trajectory (such as the soldering pattern), the next module - one-shot action template learning will be called.

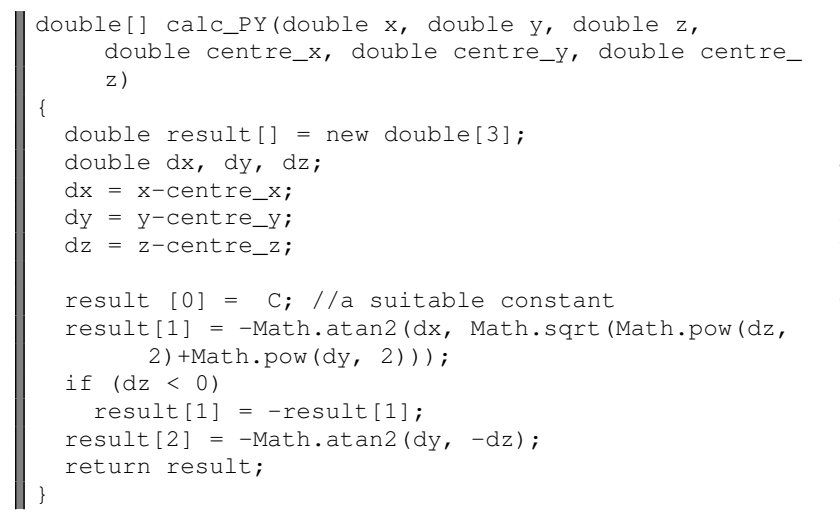

Listing 1: Algorithm to avoid KUKA inverse kinematics singularity

2) One-shot action template learning: This module records the action trajectory demonstrated by the human operator as an action template using the approach in [8] integrated with a few fine heuristic adjustments described in the following paragraphs. This is an essential step towards reusing the demonstrated action for the corresponding task in production phase where the workpiece and the task action are vastly similar in nature with requirement of minor adaptation and generalisation, such as slight deviation of workpiece size, shape, location and/or pose. Using the morphable template learning approach, we can address this issue as a template warpping problem. To achieve higher efficiency in production mode, this module screens through the action template and removes redundant movements which the human operator introduces due to hesitance, wrong movement or pause for observation, such as, prolonged duration of pause, random movements and prolonged duration of free movements. These redundant actions add to the time overhead in production which should be removed before the action template gets recorded. This step is essential to allow demonstration of task actions without prior training on specific workpieces.

As a safety feature, the action template is screened also for violation of safe movement criteria which can cause the robot to come to a halt. There are at least two contributing factors that can break the safe movement criteria: 1) The user commands the robot to move at a high speed; 2) The removal of intermediate waypoints causes either the robot to move through the object or move at a high speed. Once a violation is detected, an intermediate waypoint is introduced in the free movement zone. This process is executed until no further violation is found.

\section{B. Production Mode}

In the production mode, there are only two dependent input/output sources, namely the workpiece geometry readings and the action template database. Likewise, we will retrieve the workpiece geometry from a file and the corresponding action template from the database. Using the approach detailed in [12], we will morph the template from the associated geometry to the new production geometry to produce a new task action. As this action is a trajectory time-series, we will have to check the action against the safe movement criteria and follow the procedure described in Section III-A.2. The action is then executed by the KUKA inverse kinematics module until the successful completion of the task. If further workpiece is present either at the same location or at an alternative location, the process will continue. This will allow mass production of the same task without having the production workpieces at the same previous location. This can help to reduce the time overhead for placing and removing a new workpiece.

\section{EXPERIMENT AND RESULTS}

\section{A. Experiment Scenario}

This novel industrial robotic system is implemented for a class of industrial problem on surface contact applications, such as soldering and painting where the tool has to follow a pattern on the surface of a workpiece. In this experiment, we will install a painting brush as an extended end-effector of the KUKA LBR. A total of ten computer scientists (i.e. no prior skills of remote robot operation) are used as experimental subjects. Each subject is asked to write two characters "hi" on the designated surface strip of a red ball in three successive trials. No limits, such as duration and size, are conveyed to the subjects.

\section{B. Experimental Setup}

As network latency issue is not a research topic of this work, we set up this system implementation in a dedicated local area network where all computers are on Gigabit Ethernet connections. The overall network has a worse case transmission latency of below $1 \mathrm{~ms}$.

The experimental setup is illustrated in Fig. 4. Two slightly different spherical balls have been set up at different location and orientation with respect to the KUKA LBR as shown in Fig. 4b. The experimental subject is instructed to demonstrate the writing on the red ball while the KUKA LBR will simulate the production mode on the blue ball. The subject is blocked from direct viewing of the robot and the workspace. The subject's visual cues come solely from the large monitor which displays the global camera view of the workspace (shown in Fig. 4b) and the local camera view of the workpiece (shown in Fig. 4c). The red ball is taped with a plastic strip to provide the user with some reference as well as a designated area for painting the characters.

\section{Evaluation of Experiment}

In this work, we will consider both quantitative and qualitative evaluation of the System.

1) Simple survey question: Qualitatively, each subject will be asked for a simple survey question at the end of his/her experiment session on whether this System is easy to use. In our preliminary user studies that allow full 6DoF access to the robot's workspace, all expert users on 


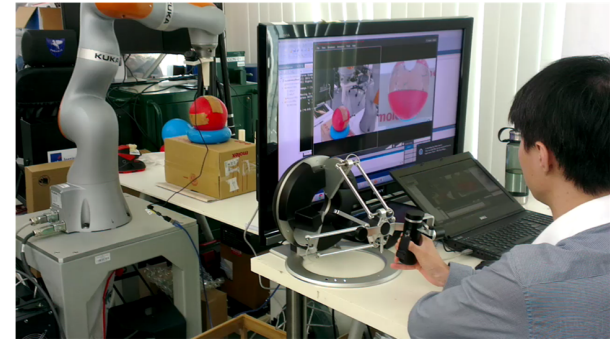

(a)

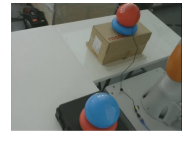

(b)

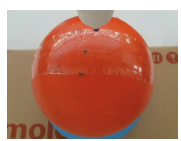

(c)
Fig. 4: The experimental setup. The subject controls the robot via the haptic device without direct view of the robot workspace as illustrated in (a). The visual feedback to the user is through the global camera view in (b) and local camera view in (c) from the large display monitor.

both the KUKA robot and the Omega-7 haptic device found it difficult to manoeuvre the robot's end-effector. Thus, we would not carry out experiments to compare systems with shared control and with full DoF access. On the quantitative side, we will investigate the following factors:

2) Training effect: If the system is not easy to use, there will be training effect when user has the chance to practise over the trials. As each human subject is asked to use the same system to perform the same task three times, the nullhypothesis of improvement over the time durations taken to complete the task is the same can be statistically tested using Analysis of Variance (ANOVA) Single Factor test.

3) Faster production mode: As we hypothesise that even the most experienced human operator will have redundant motion during his/her demonstration of an action, thus with the improved learning-by-demonstration template approach, we can make the system more efficient in the production phase. This can be tested using two-sample t-test to compare the population means between the actions executed by the human operator against the action performed by the KUKA LBR during production in all trials.

\section{Results and Discussions}

All human subjects carried out the experimental trials with the same experimental conditions. A sample of the trial is shown in Fig. 5. The human subject was asked to write the three-stroke "hi" on the red ball which sat on a much higher dais as compared to the blue ball. After the demonstration is completed, the robot switched to production mode and executed the action template on the blue ball at a different location and orientation. As we can see from the figure, the two paintings have very high similarity. As the oneshot template learning algorithm used in this work has been demonstrated to preserve the original spatial relations with a very high correlation of 0.99 [12], we shall not carry the similarity test again in this work.

Out of the 10 subjects, 8 indicated that they found the system was very easy to use, right from the first trial. However, there were two subjects finding it difficult to map their hand movement on the haptic device to the movement of the robot end-effector. They are subject numbers 2 and

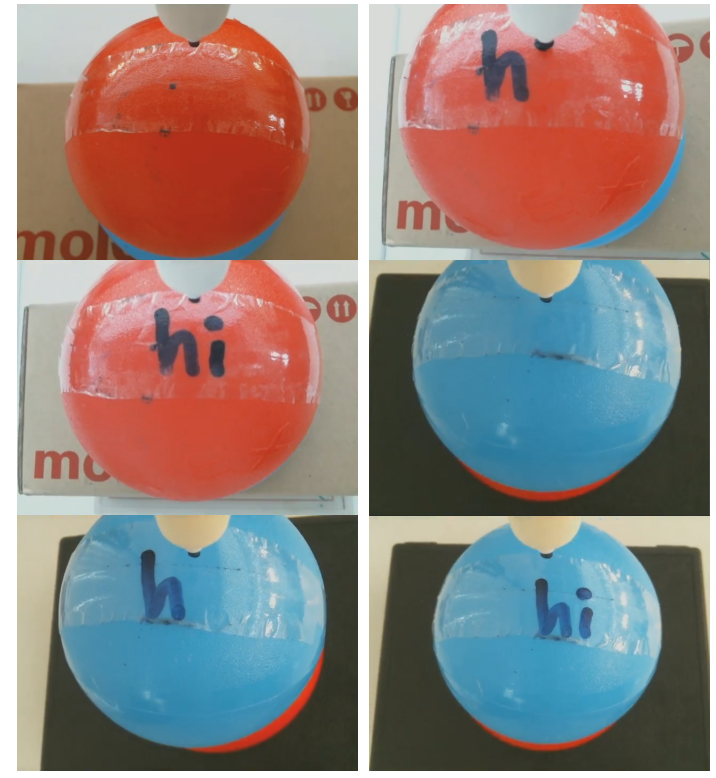

Fig. 5: An illustration of the character painting experiment. The human subject performs the demonstration on the red ball while the KUKA LBR reproduced a similar template action on the blue at a different location and orientation.

4 shown in Fig. 6. Both subjects could not figure out an easy way to control the robot throughout the trials. Subject 2 painted the surface very slowly, however when he tried to move in between strokes, he produced abrupt motions. This resulted in two characteristics in his demonstrations: 1) The duration for each trial is high without much redundancy to remove; 2) The abrupt motions in fact caused the system to introduce extra intermediate waypoints in order for the system to produce smoother movements. This resulted in slight increase in the duration in the production mode for trials 2 and 3. Subject 4 hesitated significantly trying to locate a good starting point for each stroke. This resulted in a very large disparity between the demonstration and production durations as many hesitant waypoints in the mid-air have been removed by the system.

We then applied ANOVA Single Factor analysis for the sample means of first trial and third trial to test for any effect of training. The result showed that the $\mathrm{F}$ statistics of 1.88 is significantly smaller than the F-critical statistics of 3.35. Thus, we do not reject the null-hypothesis that the sample means of 1 st trial and 3rd trial are equal. We further performed a paired two sample t-test between these two trials. The result shows that the t-stat (2.07) is between \pm the two-tail t-critical value (2.26). The observed difference between the trial sample means is unable to conclude that the average trial durations between first trial and third trial differ significantly. Thus, we are confident that the implementation of this system is easy to use. The inclusion of a local camera view and the implementation of the haptic control device have improved the situatedness of the KUKA workspace. Thus there is no training effect exhibited for this setup.

The last test performed on the experimental data is the two 


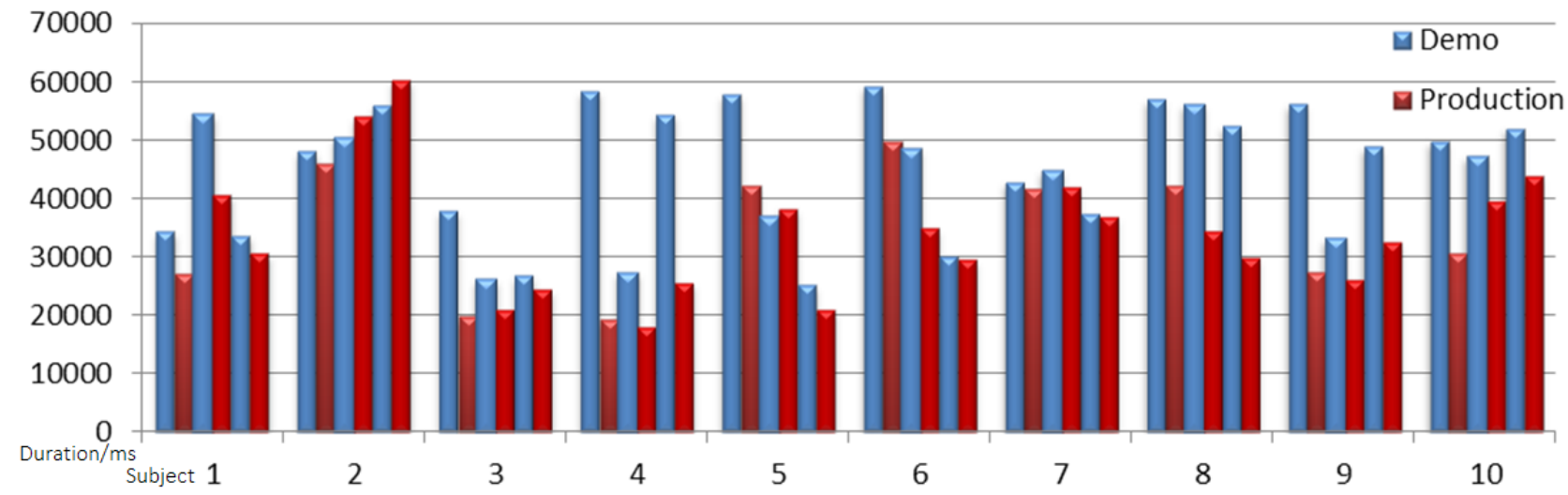

Fig. 6: The durations of all experimental trials grouped by each human subject. The blue bars are the demonstration duration performed by the human subjects while the red bars represent the reproduction of the same demonstration during the production mode executed autonomously by the robot. A total of 10 subjects participated in the experiment and each carried out 3 trials of the same task of writing the two characters "hi".

sample t-test between the durations of the demonstration and the production. Each trial is treated as an independent sample as there is minimal training effect for the subject population. The result shows that t-stat (5.46) is greater than the two-tail t-critical value (2.05) with p-value of approximately 0 . This demonstrated that the observed mean difference between the demonstration durations (mean 44.7s) and the production durations (mean 34.26s) is significantly different. We can therefore conclude that the production mode is executed almost certainly faster than the demonstration mode. This implementation of the system improves the production efficiency.

\section{CONCLUSions}

We have presented the first prototype of our novel industrial robotic system which targeted at addressing the human-robot interactivity issue for HMLV applications. This prototyped system has been implemented using a KUKA LBR iiwa 14 robot manipulator and an Omega-7 haptic device with shared control and learning by demonstration paradigms. The experiment conducted to assess the performance of the system demonstrated the merits both qualitatively and quantitatively in terms of user acceptance, efficiency and system implementation.

As the system is currently dependent on accurate input of workpiece geometry, our next step is to integrate perception and compliance control to allow the system to work more robustly. Perception can be used as an estimate of the workpiece geometry. As this estimated model is subject to unknown noise, compliance control mechanism could supplement the action execution by allowing the robot to comply to the surface of the workpiece and thus minimise the effect of noise present in the perception.

\section{ACKNOWLEDGEMENT}

This work is supported by the Science and Engineering Research Council (SERC, A*STAR, Singapore) Grant No. 1225100001 .

\section{REFERENCES}

[1] Y. Su, Y. Gao, Y. Wu, W. Dong, W. Wang, and Z. Du, "YARC - A Universal Kinematic Controller for Serial Robots based on PMAC and Movelt!," in Proceedings of the 2014 Asia-Pacific Signal and Information Processing Association Annual Submit and Conference (APSIPA ASC), 2014.

[2] S. Hassan and Y. Jan, "Design of 6-DOF Manipulator Intuitive Teaching System Using Smart Phone Orientation: User Friendly and Intuitive Teaching Operation for 6-DOF Manipulator," in Proceedings of the 2013 4th International Conference on Intelligent Systems, Modelling and Simulation, pp. 364-369, IEEE, Jan. 2013.

[3] A. Billard, S. Calinon, R. Dillmann, S. Schaal, B. Siciliano, and O. Khatib, "Robot Programming by Demonstration," in Springer Handbook of Robotics (B. Siciliano and O. Khatib, eds.), ch. 59, pp. 1371-1394, Berlin, Heidelberg: Springer Berlin Heidelberg, 2008.

[4] Y. Wu and Y. Demiris, "Learning Dynamical Representations of Tools for Tool-Use Recognition," in Proceedings of the 2011 IEEE International Conference on Robotics and Biomimetics (ROBIO), (Phuket, Thailand), pp. 2664-2669, IEEE, Dec. 2011.

[5] F. Conti, J. Park, and O. Khatib, "Interface Design and Control Strategies for a Robot Assisted Ultrasonic Examination System," in Experimental Robotics, vol. 79 of Springer Tracts in Advanced Robotics, ch. 79, pp. 97-113, Berlin: Springer, 2014.

[6] S. Calinon, F. Guenter, and A. Billard, "On Learning the Statistical Representation of a Task and Generalizing it to Various Contexts," in Proceedings of the 2006 IEEE International Conference on Robotics and Automation (ICRA), pp. 2978-2983, IEEE, 2006.

[7] M. Geravand, F. Flacco, and A. De Luca, "Human-Robot Physical Interaction and Collaboration Using an Industrial Robot with a Closed Control Architecture," in Proceedings of the 2013 IEEE International Conference on Robotics and Automation (ICRA), pp. 4000-4007, IEEE, May 2013.

[8] Y. Wu, Y. Su, and Y. Demiris, "A Morphable Template Framework for Robot Learning by Demonstration: Integrating One-shot and Incremental Learning Approaches," Robotics and Autonomous Systems, May 2014.

[9] Y. Li and S. S. Ge, "Human-Robot Collaboration Based on Motion Intention Estimation," IEEE/ASME Transactions on Mechatronics, vol. 19, pp. 1007-1014, June 2014.

[10] Y. Li and S. S. Ge, "Force Tracking Control for Motion Synchronization in Human-Robot Collaboration," Robotica, pp. 1-22, Aug. 2014

[11] Y. Li, K. P. Tee, R. Yan, D. K. Limbu, and S. S. Ge, "Shared Control of Human and Robot by Approximate Dynamic Programming," in Proceedings of the 2015 American Control Conference, (Chicago, USA), 2015.

[12] Y. Wu and Y. Demiris, "Towards One Shot Learning by Imitation for Humanoid Robots," in Proceedings of the 2010 IEEE International Conference on Robotics and Automation (ICRA), (Anchorage, United States), pp. 2889-2894, IEEE, May 2010. 